\title{
KRGÖ und FRQ im Jahr 2017
}

Am 12. Oktober 2017 beschloss die philosophisch-historische Klasse der ÖAW die Verlängerung ihrer Kommission für Rechtsgeschichte Österreichs (KRGÖ) auf unbestimmte Zeit. Damit existiert erstmals in der Geschichte der ÖAW eine nicht bloß befristet eingesetzte rechtshistorische Kommission. Der Fortgang ihrer Arbeiten wird regelmäßig evaluiert werden. Für die Funktionsperiode 1. Jänner 2018 bis 31. Dezember 2022 wurde folgende Zusammensetzung beschlossen: Ao.Univ.-Prof. DDr. Gerhard AMMERER (Salzburg), Assoz.Univ.-Prof. Dr. Elisabeth GreIF (Linz), k.M. Univ.-Prof. Dr. Gabriele HAUG-MORITZ (Graz), o.Univ.-Prof. DDr. Herbert KALB (Linz), ao.Univ.-Prof. Dr. Gerald KOHL (Wien), ao.Univ.-Prof. Dr. Christian NeschWARA (Wien), w.M. ao.Univ.-Prof. Dr. Thomas OlechowsKi (Wien), Univ.-Ass. Dr. Eva OrtLieb (Graz), ao.Univ.-Prof. Dr. Ilse ReITERZATLOUKAL (Wien), Univ.-Prof. DDr. Martin SCHENNACH (Innsbruck), k.M. Univ.-Prof. Dr. Arno STROHMEYER (Salzburg), em.o.Univ.-Prof. Dr. Gunter WESENER (Graz), w.M. Univ.-Prof. Dr. Ewald WiEDERIN (Wien), w.M. Univ.-Prof. Dr. Dr.h.c. Thomas WinKelbaUeR (Wien), ao.Univ.-Prof. Dr. Anita ZIEGERHOFER (Graz).

Gemeinsam mit der Forschungsstelle für Rechtsquellenerschließung (FRQ) der Universität Wien brachte die KRGÖ im Jahr 2017 einen neuen Band mit Rechtsquellen in der Reihe „Fontes iuris" heraus. Dieser Band trägt die Nummer 25 und enthält die Rechtsquellen der Stadt Hartberg in der Steiermark, herausgegeben von Alois KeRnBAUER. Das 548 Seiten starke Buch deckt einen Zeitraum von knapp sieben Jahrhunderten (1128-1826) ab, hat jedoch einen deutlichen Schwerpunkt im 17. Jahrhundert, als Schloss und Stadt Hartberg infolge der notorischen Geldknappheit der Habsburger von diesen an die Grafen von Paar verkauft wurden, in deren Hand die Stadtherrschaft bis zur Grundentlastung Mitte des 19. Jahrhunderts verblieb was schwerwiegende, negative Folgen für die Entwicklung der Stadt hatte.

Von der Publikationskommission der ÖAW angenommen wurden die Manuskripte zu den Fontes iuris-Bänden 26 (Die Tiroler Landesordnungen) und 10/5 (Die Wiener Stadtbücher, Teil 5), deren Drucklegung Anfang 2018 erfolgt.

Am 3. Oktober 2017 wurde im Rahmen einer Veranstaltung der Wiener Rechtsgeschichtlichen Gesellschaft die Monographie „Das Dienst-, Habilitations- und Disziplinarrecht der Universität Wien 1848-1938“ von Univ.-Ass. Dr. Kamila STAUDIGL-CIECHOWICZ LL.M. präsentiert.

Am 16. und 17. Oktober fand am Juridicum der Universität Wien die Tagung „Normsetzung im Notstand. Außerordentliche Gesetzgebungsbefugnisse im 19. und 20. Jahrhundert" statt, die von KRGÖ und FRQ gemeinsam organisiert und vom Dekan der Rechtswissenschaftlichen Fakultät gemeinsam mit dem Präsidenten des Verfassungsgerichtshofes eröffnet wurde. Anlass war der 100. Jahrestag des Inkrafttretens des Kriegswirtschaftlichen Ermächtigungsgesetzes vom 24. Juli 1917. Sowohl dieses Gesetz als auch die anderen historischen und aktuellen Notverordnungsrechte in Österreich wurden kritisch analysiert und mit vergleichbaren Phänomenen in Deutschland, Italien, Jugoslawien Polen, der Tschechoslowakei und Ungarn verglichen. Die 
Drucklegung der Referate wird im BRGÖ-Band 2018/2 erfolgen.

Das Projekt „St. Germain“, an dem außer KRGÖ und FRQ auch die rechtshistorischen Institute der Universitäten Graz und Linz beteiligt sind, hat in einem ersten wichtigen Arbeitsschritt einen umfassender Textvergleich des 1919 mit Österreich abgeschlossenen Vertrages mit jenen Verträgen, die mit Deutschland zu Versailles, mit Ungarn zu Trianon, mit der Türkei zu Sèvres und mit Bulgarien zu Neuilly geschlossenen wurden, durchgeführt. Über weite Strecken wurden Parallelitäten, jedoch auch markante Abweichungen festgestellt; die Unterschiede variieren von Vertrag zu Vertrag beträchtlich. Für die eigentliche Kommentierung des Vertrages wurde ein zehnköpfiges Team gebildet, das sich am 4. Mai zu einem Workshop am Institut für Neuzeit- und Zeitgeschichtsforschung der ÖAW traf, um die Arbeitsabläufe zu bündeln und offene Fragen zu besprechen. Im Anschluss daran wurde mit umfassenden Archivrecherchen, zunächst in österreichischen Archiven, begonnen.

Im Personalstand der FRQ fanden einige Änderungen statt: Frau Katharina BERNOLD kehrte mit 13. Februar, Frau Dr. Kamila STAUDIGLCIECHOWICZ mit 30. September 2017 aus der
Elternkarenz zurück. Frau Mag. Laura Rosemarie RATHMANNER schied mit 29. Juli 2017 aus dem Personalstand der Universität Wien aus und trat mit 1. August 2017 als FWF-finanzerte Projektmitarbeiterin für das oben genannte Projekt "St. Germain" in den Personalstand der Universität Linz.

Die FRQ verbrauchte im Berichtszeitraum Personal- und Sachmittel der Universität Wien in Höhe von $€ 115.953,63$. Die KRGÖ erhielt von der ÖAW Sachmittel in Höhe von $€$ 8.973,05 (zusammengesetzt aus dem ordentlichen Budget, einer Sonderdotation für die Oktober-Tagung, einer Förderung der Publikation des Fontes iuris-Bandes 25 aus Mitteln der Politzer-Stiftung sowie der Finanzierung des Lektorats der BRGÖ). Im Rahmen des Projekts zur ErschlieBung der Reichshofratsakten kamen $€$ 35.509,92 von der Akademie der Wissenschaften zu Göttingen. Vom FWF kamen insgesamt $€$ 12.453,70 der FRQ zugute. Sonstige Drittmittelgeber unterstützten die Drucklegung des Fontes iurisBandes mit $€$ 6.300,-. Das Gesamtbudget beider Forschungseinheiten setzte sich somit zu $65,19 \%$ aus Mitteln der Universität Wien, zu 5,04 \% aus Mitteln der ÖAW und zu 29,77\% aus Drittmitteln zusammen.

Thomas OLECHOWSKI 
